\title{
La phaeoramulariose des agrumes au Cameroun due à Phaeoramularia angolensis : expression parasitaire à différentes altitudes
}

Jean Kuate ${ }^{a \star}$, Eric Fouréb, Jacob Fokoc ${ }^{c}$ Daniel Duceliera ${ }^{a}$ Félix Tchio ${ }^{d}$

\author{
a Irad Nkolbisson, \\ Programme Fruits, \\ BP 2067, Yaoundé, \\ Cameroun \\ irad-fruits@camnet.cm \\ b Carbap Nyombé, \\ BP 832, Douala, \\ Cameroun \\ c Université de Dschang / \\ Carfop, \\ BP 409, Dschang, \\ Cameroun \\ d Irad, \\ BP 13, Nyombé, \\ Cameroun
}

* Correspondance et tirés à part

Reçu le 22 novembre 2001 Accepté le 25 mars 2002

Fruits, 2002, vol. 57, p. 207-218 (C) 2002 Cirad/EDP Sciences All rights reserved

DOI: 10.1051/fruits:2002018

RESUMEN EsPañol, p. 218

\section{Citrus phaeoramulariosis due to Phaeoramularia angolensis in Cameroon: parasitic expression at different altitudes.}

Abstract - Introduction. Citrus leaf and fruit spot due to Phaeoramularia angolensis [De Carvalho \& Mendes] P.M. Kirk represents a serious production constraint in tropical Africa. Disease expression may vary greatly from one location to another. Materials and methods. Disease development was studied on young seedlings of grapefruit (Marsh cultivar) inoculated with a conidia suspension and transferred to eight locations at (80 to 1250) m elevation. Temperature $(\mathrm{T})$ and relative humidity $(\mathrm{RH})$ were recorded on the sites during the work. Results and discussion. Disease incidence increased with altitude. The Nyombe Station (80 $\mathrm{m}$ elevation, Taverage $>25^{\circ} \mathrm{C}$ with Tmax above $30^{\circ} \mathrm{C}$ and RHaverage $<80 \%$, with RHmin often below 60\%) was far less conducive to disease development than the rest of the locations, which experienced cooler and more humid climatic conditions (Tmin $<20{ }^{\circ} \mathrm{C}$ and RHmin $>60 \%$ ). Conclusion. This study provides preliminary information on characterization of regions that may be invaded by $P$. angolensis. The work should be pursued, including different ecological situations, to enable the mapping of the risk of this threat.

Cameroon / Citrus paradisi / fungal diseases / Pbaeoramularia angolensis / blotches / symptoms / site factors / altitude / temperature / relative humidity

\section{La phaeoramulariose des agrumes au Cameroun due à Phaeoramularia angolensis : expression parasitaire à différentes altitudes.}

Résumé - Introduction. La phaeoramulariose ou cercosporiose des agrumes due à Phaeoramularia angolensis (De Carvalho \& Mendes) P.M. Kirk constitue une sérieuse contrainte de production en Afrique tropicale. L'expression parasitaire peut varier de façon notable d'une localité à l'autre. Matériel et méthodes. L'évolution de la maladie sur de jeunes plants de pomelo Marsh inoculés à l'aide d'une suspension conidienne a été étudiée sur huit sites d'altitudes différentes $(80 \mathrm{~m}$ à $1250 \mathrm{~m}$ ). Les données de température $(\mathrm{T})$ et d'humidité relative (HR) maximales, moyennes et minimales ont été relevées sur les sites durant la période d'observation. Résultats et discussion. L'incidence de la maladie a varié de façon croissante en fonction de l'altitude. Sur les huit sites étudiés, celui de Nyombé à $80 \mathrm{~m}$ d'altitude (Tmoy $>25{ }^{\circ} \mathrm{C}$, Tmax $>30^{\circ} \mathrm{C}$ avec HRmoy $<80 \%$, HRmin < $60 \%$ ) s'est révélé très défavorable à la maladie. En revanche, l'expression des symptômes a été complète sur les autres sites qui présentent des conditions climatiques plus fraîches et plus humides (Tmin $<20{ }^{\circ} \mathrm{C}$ et HRmin $>60 \%$ ). Conclusion. Ces résultats constituent les premières données de caractérisation des zones à risque d'invasion par $P$. angolensis. Ces résultats joints à d'autres travaux ultérieurs impliquant différents contextes écologiques permettront d'établir une cartographie du risque épidémique.

Cameroun / Citrus paradisi / maladie fongique / Pbaeoramularia angolensis / cercosporiose / symptôme / facteur lié au site / altitude / température / humidité relative 


\section{Introduction}

La cercosporiose des agrumes a été signalée pour la première fois en Angola et au Mozambique [1, 2]. Précédemment décrit comme Cercospora angolensis (De Carvalho \& Mendes), l'agent pathogène est actuellement connu sous le nom de Phaeoramularia angolensis (De Carvalho \& Mendes) P.M. Kirk [3]. Cette maladie attaque à la fois les feuilles et les fruits provoquant de nombreuses lésions. Les pertes de récolte peuvent varier de (50 à 100) \%.

Un certain nombre de travaux de recherche portant sur l'étude de la sensibilité variétale et le contrôle de la maladie ont été réalisés, principalement au Cameroun et au Kenya. Les premières observations en collections d'agrumes au Cameroun ont permis de noter la sensibilité de plusieurs cultivars [4-6]. Plus d'une centaine de variétés d'agrumes ont ainsi été évaluées sans déceler de résistance prononcée. Par ailleurs, au cours d'essais d'utilisation de fongicides, l'efficacité des benzimidazoles et des produits cupriques a été mise en évidence, mais non celle des triazoles [7-9].

La description détaillée des symptômes a été un autre point important des travaux entrepris sur la cercosporiose [10-12]. La lésion foliaire se caractérise par une zone centrale circulaire de tissus desséchés, bordée d'une couronne brune, le tout entouré d'un halo jaune. Parfois, le centre de la nécrose peut se perforer et former un trou rond. Sur jeunes fruits, la maladie commence par le développement d'un mamelon dont le sommet brunit et s'affaisse en un cratère où se développe par la suite un duvet gris noir constitué de conidiophores porteurs de nombreuses conidies. En revanche, il n'apparaît pas de mamelon sur fruits âgés mais des lésions brunes entourées d'un halo jaune. Il arrive parfois que le fruit éclate au niveau de la lésion.

L'étude du développement de la maladie (incidence et sévérité) a été réalisée en zone d'altitude élevée [13] et en zone forestière humide de moyenne altitude [14, 15]. Des attaques particulièrement sévères sur les jeunes organes (feuilles et fruits) ont ainsi pu être notées en saison des pluies.
Des floraisons décalées dues à un arrosage des arbres en saison sèche ont permis de réduire significativement les attaques de la maladie [14]. En zone forestière humide du Cameroun, l'incidence de la maladie est beaucoup plus forte entre septembre et novembre (seconde saison des pluies) qu'entre mars et juin (première saison des pluies). Les fruits demeurent sensibles durant tout leur développement alors que les feuilles ne sont plus réceptives à l'agent pathogène (5 à 8 ) semaines après éclatement des bourgeons.

Bien qu'aucune étude détaillée n'ait encore porté sur cet aspect, il a été fréquemment signalé que la maladie était d'autant plus grave que l'altitude était élevée [ 4 , $7,8,12,13,16]$. Les premières inoculations réalisées à la station de Nyombé (80 m d'altitude) n'ayant pas permis d'observer une expression complète des symptômes de la maladie [17], il paraissait intéressant d'observer les plants dans d'autres contextes écologiques. Nous avons donc cherché à préciser ces observations en étudiant l'évolution de la maladie sur de jeunes plants de pomelo greffés sur Citrus volkameriana, inoculés et plantés sur des sites d'altitudes différentes. Les résultats ont pu être discutés en prenant en compte les données de température et d'humidité relative relevées sur les sites concernés afin d'identifier les conditions favorables au développement de la maladie sur feuilles.

\section{Matériel et méthodes}

\subsection{Les sites expérimentaux}

La zone de Nyombé a un climat de type équatorial à deux saisons : une saison des pluies de mi-mars à mi-novembre et une saison sèche de mi-novembre à mi-mars. Les moyennes annuelles de température sont proches de $27^{\circ} \mathrm{C}$ et celles de la pluviométrie peuvent atteindre $(2000$ à 3000$) \mathrm{mm}$. C'est une région volcanique aux sols riches et légers. La station d'Ekona $(400 \mathrm{~m})$ se trouve au pied du Mont Cameroun, sur la façade atlantique ; les conditions climatiques sont beaucoup plus fraîches et 
humides que celles de Nyombé. Outre les stations de Nyombé et Ekona, l'étude a concerné six sites expérimentaux implantés à différentes altitudes : Penja $(200 \mathrm{~m})$, Mélong I (700 m), Mélong II $(900 \mathrm{~m})$, Mélong III (1000 m), Mélong IV (1100 m) et Mbouroukou $(1250 \mathrm{~m})$. La température (figure 1) et l'humidité relative (figure 2), deux données climatiques susceptibles d'influencer le développement de la cercosporiose, ont été relevées sur ces différents sites durant la période d'observation des plants.

\subsection{Préparation du matériel}

\subsubsection{Matériel végétal}

Pour chaque site, huit plants de pomelo Marsh âgés de 1 an et greffés sur Citrus volkameriana ont été préparés dont cinq plants inoculés et trois plants non inoculés choisis comme témoins. Trois semaines avant la date des inoculations, les plants en pépinière ont été taillés et fertilisés par apport de 5 g d'urée dans chaque pot. De jeunes feuilles tendres et de coloration vertclair se sont alors développées.

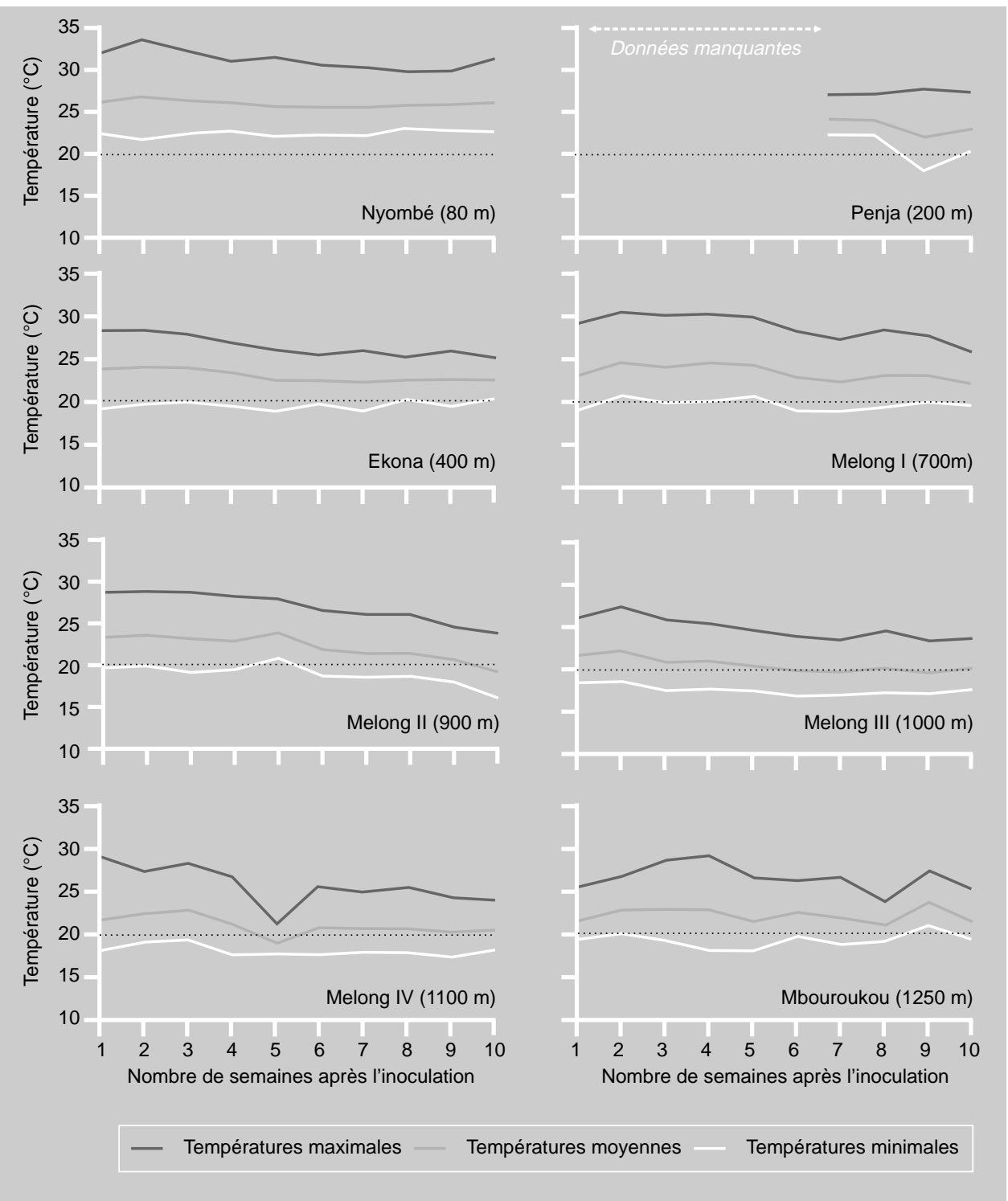

Figure 1.

Évolution des températures pendant les dix semaines qui suivent la contamination (29 mai 1995) de jeunes plants d'agrumes avec un inoculum de spores de Phaeoramularia angolensis. Observations faites sur huit sites d'observation localisés à des altitudes différentes (Cameroun). 
Figure 2.

Évolution de l'humidité relative pendant les dix semaines qui suivent la contamination (29 mai 1995) de jeunes plants d'agrumes avec un inoculum de spores de Phaeoramularia angolensis. Observations faites sur huit sites d'observation localisés à des altitudes différentes (Cameroun).
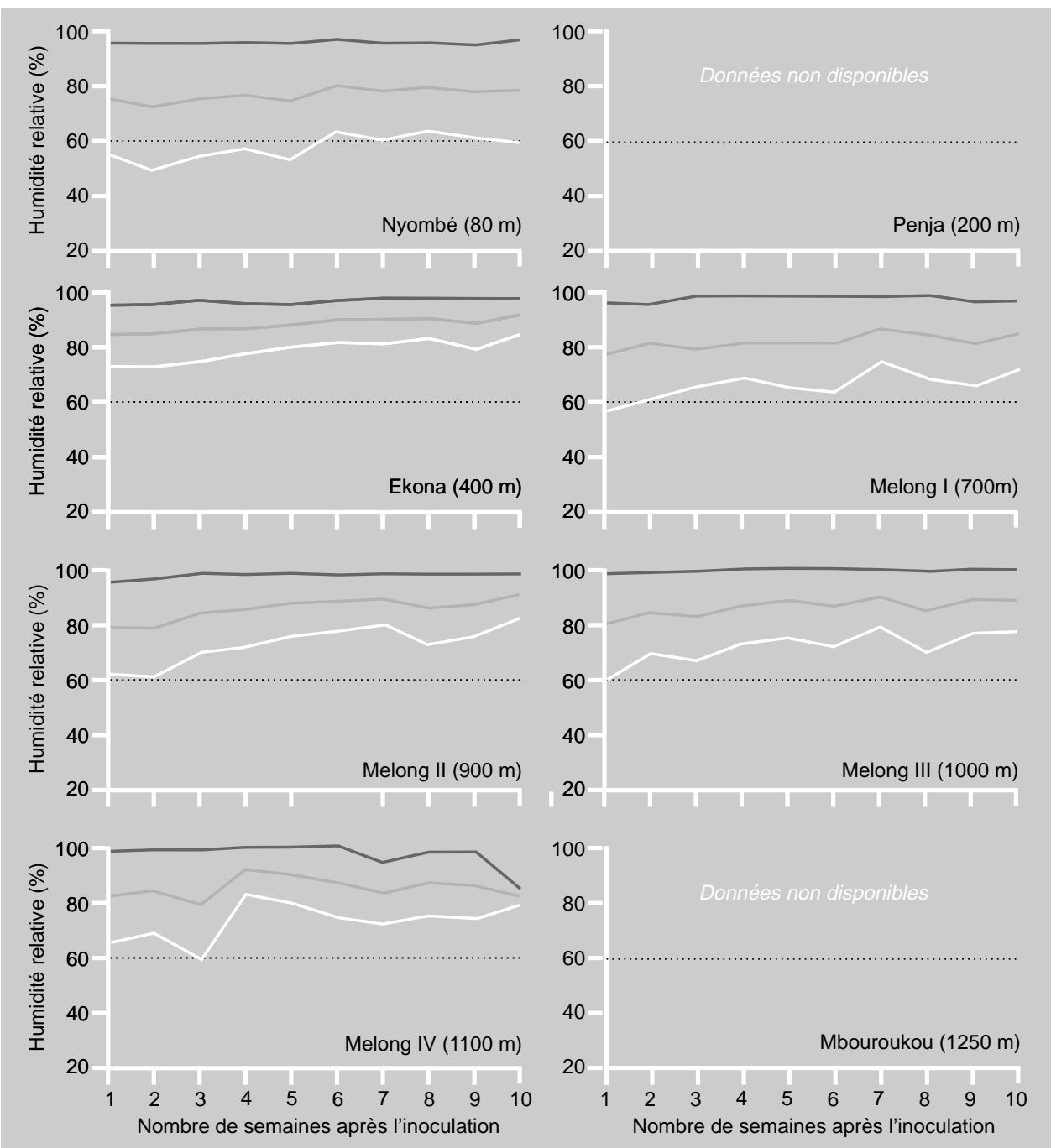

_ Humidité relative maximale _- Humidité relative moyenne __ Humidité relative minimale

\subsubsection{Matériel fongique}

Des étalements mycéliens ont été réalisés sur milieu V.8 modifié [18] en boîtes de Petri de $90 \mathrm{~mm}$ à partir d'un isolat cloné de $P$. angolensis issu d'un oranger Hamlin. Cet isolat utilisé lors de travaux antérieurs avait permis de reproduire les symptômes de cercosporiose sur toutes les feuilles de pomelo inoculées [19]. Les boîtes scellées au parafilm ont été incubées à $22{ }^{\circ} \mathrm{C}$ en lumière continue pendant $10 \mathrm{j}$ pour favoriser la production de conidies. Une suspension conidienne calibrée dans de la gélatine à $2 \%$ contenant $6 \times 10^{5}$ conidies $\times \mathrm{mL}^{-1} \mathrm{a}$ alors été préparée pour constituer l'inoculum.

\subsection{Inoculation et observation des plants}

Un certain nombre de protocoles d'inoculations artificielles de $P$. angolensis ont été rapportés dans la littérature : utilisation d'une suspension conidienne dans des extraits de peau d'orange [20] ; mélange de fragments mycéliens et de conidies en 
suspension dans de l'eau, appliqué au pinceau ou injecté à la seringue [21] ; gouttes de suspension conidienne déposées à la face inférieure des feuilles [19] ; filtrats de culture du champignon [22] ; suspensions conidiennes pulvérisées sur des feuilles détachées et mises en survie en boîte de Petri [23]. Lors de la présente étude, il a été choisi d'appliquer l'inoculum au pinceau à la face inférieure des feuilles.

Sur chacun des cinq plants expérimentaux d'un site donné, deux rameaux ont été repérés à l'aide d'une étiquette. Les inoculations ont été effectuées avec l'inoculum préparé et dosé comme mentionné ci-dessus sur les quatre premières feuilles numérotées de la base vers l'apex de chacun de ces rameaux. Les feuilles des trois plants témoins ont reçu une solution de gélatine à $2 \%$ également appliquée au pinceau à la face inférieure. Les arbres ont été plantés en ligne à $6 \mathrm{~m}$ d'intervalle et sur butte avec le collet en position haute.

Les feuilles marquées ont été observées deux fois par semaine, les mardis et vendredis, et pendant une période de 10 semaines. Durant ces observations, le nombre de lésions éventuellement apparues a été noté, ainsi que le stade de la lésion la plus évoluée $($ stade +$)$. Différents autres paramètres ont été étudiés :

- Taux de feuilles malades : chaque semaine, le nombre de feuilles malades a été compté. Les résultats ont été exprimés sous forme de pourcentages cumulés de feuilles malades par rapport à l'ensemble des feuilles observées.

- Nombre moyen de lésions par feuille : chaque semaine le nombre de lésions de différents stades a été compté. Les quatre stades d'évolution des symptômes foliaires ont été considérés : stade 1, apparition d'un petit point décoloré ; stade 2, brunissement de la lésion et apparition d'un halo jaune ; stade 3, accroissement de la lésion centrale jusqu'à atteindre (3 à 4) mm ; stade 4, dessèchement du centre de la nécrose pouvant entraîner une perforation du limbe. Le total de lésions pour chaque feuille a donc correspondu à la somme des lésions ayant atteint l'un des quatre stades. Au terme des observations, les moyennes ont été calculées pour chaque site.
- Taux de feuilles malades tombées : Chaque semaine, le nombre de feuilles tombées à la suite des attaques de cercosporiose a été noté. Ce résultat a ensuite été exprimé en pourcentage cumulé du nombre total de feuilles observées sur chaque site.

- À la fin des observations, en connaissant les dates d'apparition des différents symptômes sur feuilles, certains autres paramètres ont pu être déterminés, dont la durée minimale (durée entre la date d'inoculation et la date d'apparition du premier symptôme à un stade donné) et la durée moyenne (moyenne calculée sur l'ensemble des feuilles observées) en jours de quelques phases d'évolution des symptômes de la maladie : temps écoulé entre l'inoculation (= date 0$)$ et l'apparition du stade 1 ; entre le stade 1 et le stade 2 ; entre le stade 2 et le stade 3 ; entre le stade 3 et le stade 4 ; entre le stade 1 et la chute de la feuille.

Pour calculer les moyennes de ces différents temps, seules ont été prises en compte les feuilles qui se sont maintenues sur l'arbre pendant toute la durée d'observation. Les feuilles éventuellement tombées sans lésion de cercosporiose ont été exclues des comptes. Celles portant des lésions avant la chute ont été comptabilisées dans la catégorie "chute de feuilles malades". Une analyse de variance a été réalisée pour comparer les durées des différentes phases de la maladie.

\section{Résultats et discussion}

\subsection{Facteurs climatiques}

Les relevés effectués pendant la durée de l'expérimentation ont montré que les températures minimales, très peu variables, étaient inférieures à $20^{\circ} \mathrm{C}$ pour tous les sites exceptés ceux de Nyombé et Penja (figure 1). Par ailleurs, les températures maximales n'ont pas excédé $30^{\circ} \mathrm{C}$, sauf sur le site de Nyombé. Tous les autres sites ont eu des températures moyennes comprises entre (20 et 25$)^{\circ} \mathrm{C}$, les plus basses correspondant aux sites de Mélong III et Mélong IV. Les fluctuations les plus marquées ont été observées au site de Mélong IV (1100 m). 
L'humidité relative minimale a toujours été supérieure à $60 \%$, sauf à Nyombé (figure 2) ; des valeurs particulièrement élevées d'humidité relative minimale (> $80 \%$ ) ont été enregistrées sur le site d'Ekona. Les valeurs les plus basses de l'humidité relative maximale, qui a très peu varié durant la période d'observation, ont été observées à Nyombé. L'humidité relative moyenne a partout été supérieure à $80 \%$, sauf à Nyombé. Les plus fortes variations ont encore été notées dans le cas du site de Mélong IV.

\subsection{Expression de la cercosporiose}

Tous les plants témoins non inoculés sont restés sains durant toute la période d'observation et n'ont pas connu de chute de feuilles.

\subsubsection{Taux de feuilles malades en fonction du temps}

Une évolution particulière des symptômes a été observée sur les sites de Nyombé et de Penja. Certaines décolorations observées sur feuilles n'ont pas évolué en nécroses ; elles semblent s'être résorbées, la feuille exprimant une sorte de "rétablissement " en reprenant sa coloration normale.

C'est ainsi que sur le site de Nyombé $(80 \mathrm{~m})$, le pourcentage de feuilles malades a augmenté, atteignant près de $80 \%$ dès la deuxième semaine et ce taux a par la suite progressivement baissé, diminuant presque de moitié vers la sixième semaine. Au total, près de $60 \%$ de feuilles étaient encore saines à la fin des observations (figure 3).

Sur le site de Penja (200 m), la situation a été plus ou moins similaire ; toutefois la baisse observée après la deuxième semaine a été beaucoup moins marquée, près de $80 \%$ des feuilles étant restées malades jusqu'à la fin des observations (figure 3).

Une étude en conditions contrôlées de température et d'hygrométrie pourrait permettre de mieux comprendre les résultats d'inoculation obtenus. En effet, en condition de laboratoire, certains travaux [24] ont permis de constater que les infections sur feuilles étaient influencées par une forte concentration d'inoculum et des températures proches de $25^{\circ} \mathrm{C}$, la période de forte hygrométrie dépassant $24 \mathrm{~h}$.

Au terme de nos observations, les conditions d'hygrométrie de Nyombé (maxima $<100 \%$, moyennes < $80 \%$ et minima $<60 \%$ ) ainsi que des températures moyennes élevées $\left(>25^{\circ} \mathrm{C}\right)$ sont apparues peu favorables à l'expression de la maladie. Sur tous les autres sites où la situation inverse a été observée pour ces paramètres climatiques, la maladie s'est normalement développée sur la totalité des feuilles inoculées. À noter que parmi les décolorations observées sur feuilles à Nyombé, quelques unes, bien que rares, ont finalement pu évoluer en véritables lésions nécrotiques (stade 2).

\subsubsection{Nombre moyen de lésions par feuille en fonction du temps}

Sur les sites de Nyombé et de Penja, le nombre moyen de lésions par feuille a augmenté jusqu'à atteindre un peu moins de 20 lésions en 3 semaines sur le site de Nyombé et en 5 semaines sur celui de Penja, puis ce nombre a diminué jusqu'à moins de 15 lésions par feuille (figure 4).

Au site d'Ekona, ces moyennes ont dépassé 20 lésions par feuille dès la quatrième semaine. À partir de l'altitude du site de Mélong III (1000 m), le seuil de (20 à 25) lésions par feuille a été atteint, les valeurs les plus élevées (près de 35 lésions par feuille) ayant été obtenues à Mélong IV (1100 m). Pour l'ensemble des sites, hors Nyombé et Penja, le maximum de lésions par feuille a été atteint entre la sixième et la huitième semaine. Le plus grand nombre de lésions par feuille a été comptabilisé sur les feuilles du site de Mélong IV (figure 4) qui correspond par ailleurs aux variations thermiques et hygrométriques les plus marquées.

La baisse du nombre moyen de lésions par feuille observée à Nyombé et à Penja a résulté de la disparition des taches décolorées (premiers symptômes de la maladie). En revanche, sur les autres sites, l'augmentation constatée jusqu'à la sixième ou la huitième semaine du nombre de lésions sur les feuilles indiquerait l'apparition progressive de nouvelles lésions jusqu'à ces dates. Ces observations confirment certains résultats antérieurs [13]. Les feuilles témoins sont 


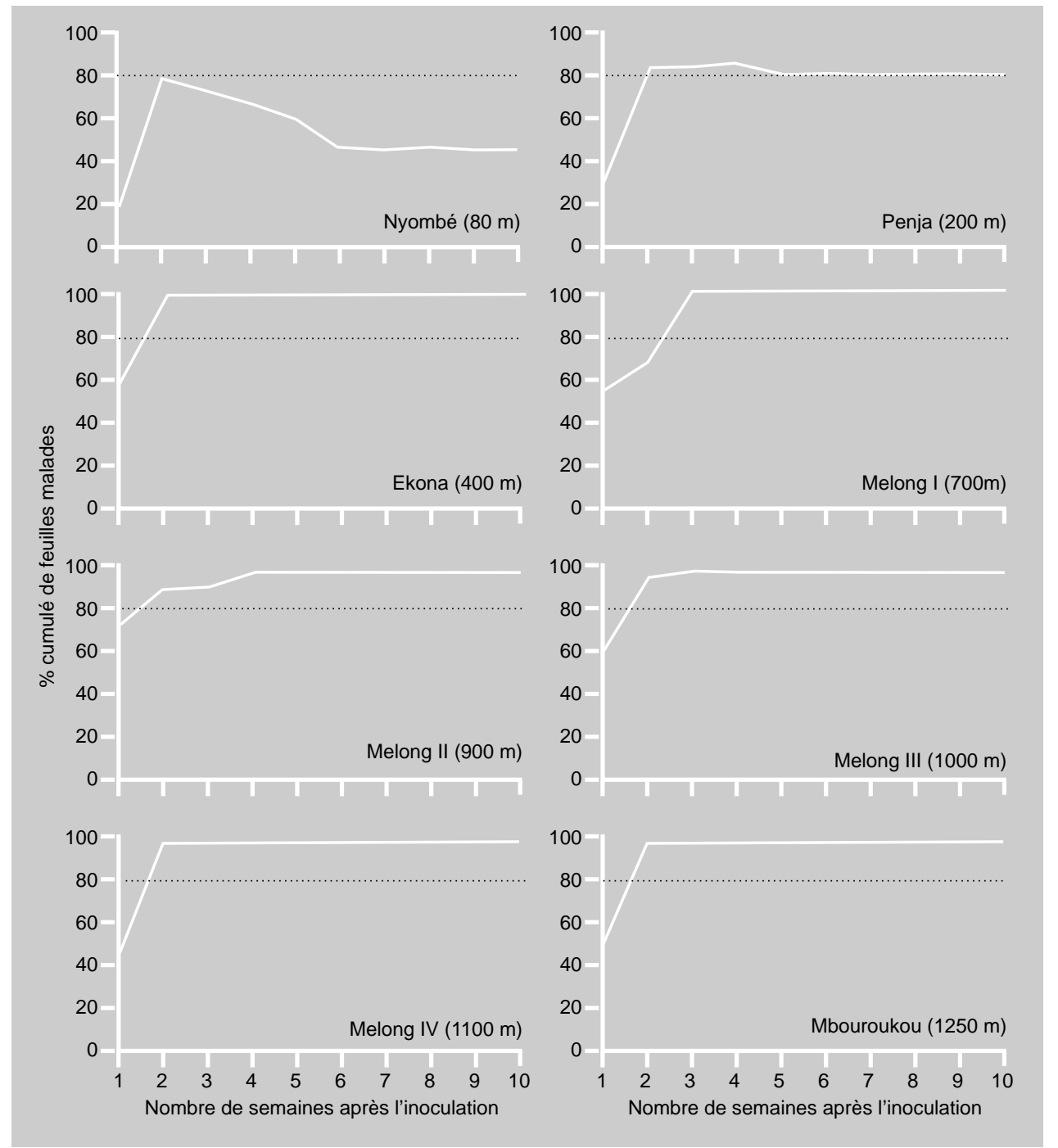

restées saines jusqu'à la fin des observations.

Lors de nos expérimentations, certaines feuilles ont présenté un jaunissement rapide et généralisé même avec un nombre relativement faible de lésions, puis elles ont chuté très rapidement (surtout sur les sites d'Ekona, de Mélong III et de Mbouroukou). Cette apparition des symptômes de jaunissement rapide pourrait être due à la technique d'inoculation au pinceau, car elle n'a pas été observée lorsque l'inoculum était déposé en gouttes sur les feuilles [19].

\subsubsection{Chutes de feuilles malades en fonction du temps}

Sur le site de Nyombé, aucune chute de feuille malade n'a été observée. Les chutes de feuilles sont restées relativement faibles (moins de $20 \%$ ) pour les sites de Penja, Mélong I et Mbouroukou. Sur les sites d’Ekona, Mélong II, Mélong III et Mélong IV, les chutes de feuilles ont atteint ou dépassé $50 \%$, les plus nombreuses (> $60 \%$ ) correspondant au site de Mélong IV (figure 5).

L'absence de chute de feuilles malades au site de Nyombé pourrait être expliquée
Figure 3.

Évolution du taux de feuilles malades de jeunes plants d'agrumes pendant les dix semaines qui suivent leur contamination (29 mai 1995) avec un inoculum de spores de Phaeoramularia angolensis. Observations faites sur huit sites d'observation localisés à des altitudes différentes (Cameroun). 
Figure 4.

Évolution du nombre moyen de lésions par feuilles de jeunes plants d'agrumes pendant les dix semaines qui suivent leur contamination (29 mai 1995) avec un inoculum de spores de Phaeoramularia angolensis. Observations faites sur huit sites d'observation localisés à des altitudes différentes (Cameroun).

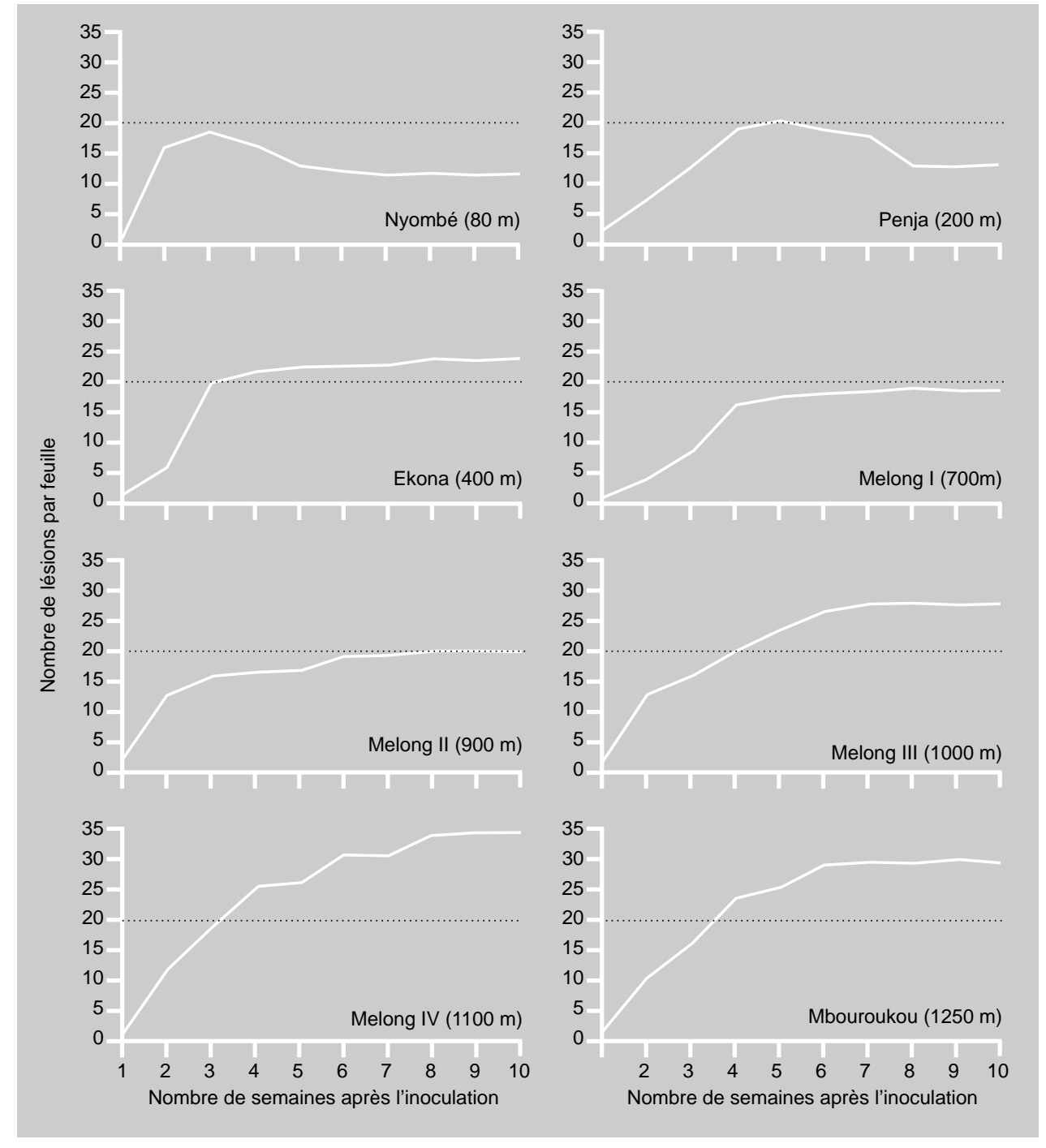

par le niveau extrêmement faible de l'expression parasitaire en ces lieux (lésions bloquées aux stades 1 et 2). En revanche, les nombreuses chutes enregistrées pour les plants des autres sites seraient le résultat d'un niveau élevé d'évolution de la maladie. En effet, il existerait une forte corrélation entre le nombre moyen de lésions et les chutes de feuilles qui en résultent [13].

\subsubsection{Durée des différentes phases des symptômes de la maladie}

L'effet du site expérimental a été hautement significatif pour la durée d'incubation de la maladie développée sur les feuilles inoculées (tableau I).

La durée d'incubation la plus courte a été observée à Ekona. Le temps de transition entre le stade 1 du développement de la cercosporiose et le stade 2 a été aussi plus court à Ekona (environ $10 \mathrm{j}$ ) que sur les autres sites. Les durées d'incubation les plus longues ont été enregistrées à Nyombé (41 j), Mélong II (32 j), Mélong IV (32 j) et Mbouroukou (environ $37 \mathrm{j}$ ). Au site de Nyombé, les lésions n'ont pas évolué audelà du stade 2. Aucune différence significative n'a pu être mise en évidence entre 


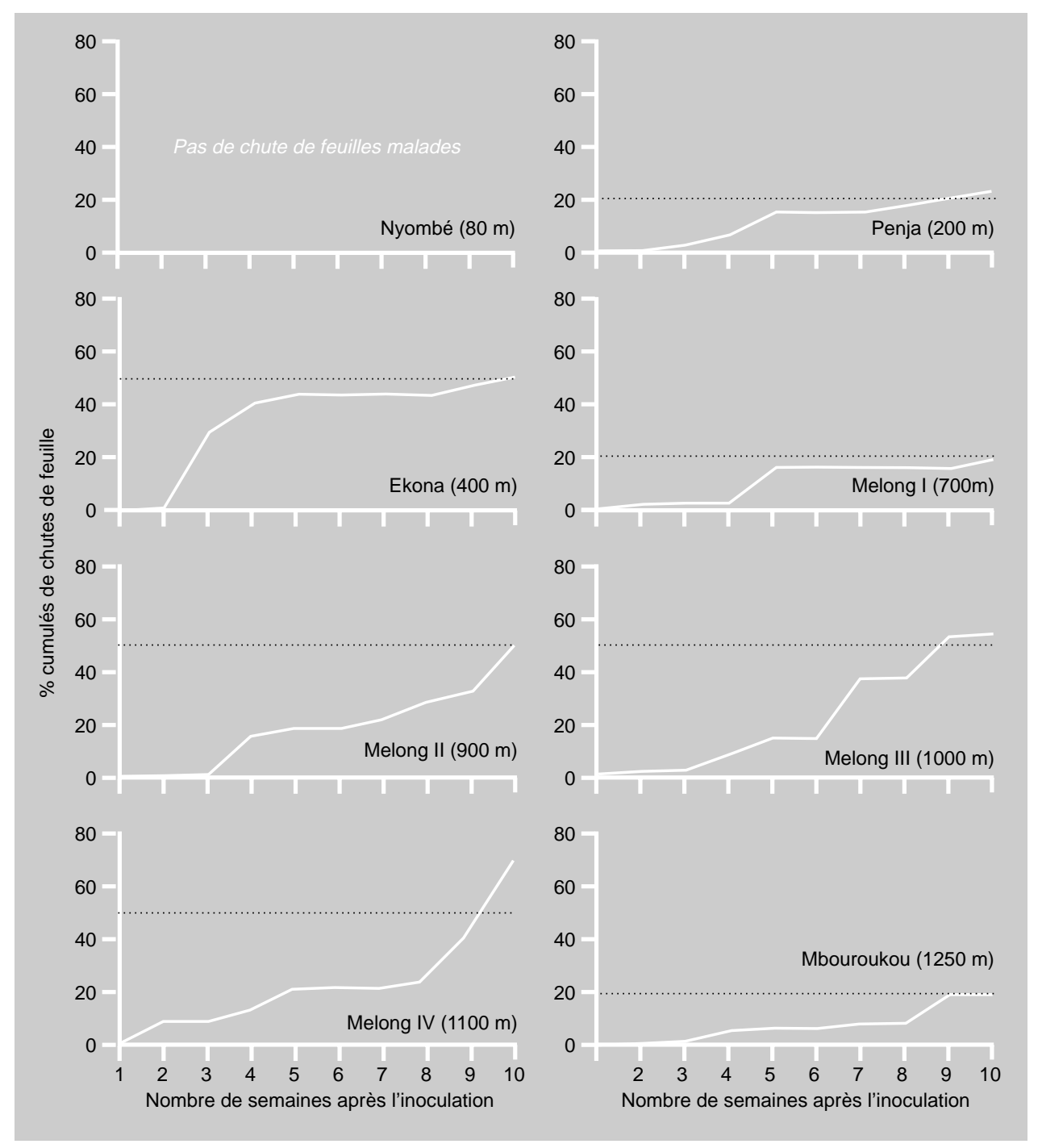

les sites quant à la durée de transition entre les stades 2 et 3 (tableau I).

Entre les stades 3 et 4 , la durée la plus longue (42 j) a été obtenue à Mbouroukou, alors qu'à Mélong I, cette phase a été beaucoup plus courte.

L'évolution complète des symptômes de la maladie du stade 1 au stade 4 a été significativement la plus longue à Mbouroukou et la plus courte à Ekona.

La défoliation totale a été obtenue en 44 j après l'inoculation au site de Mélong II, alors qu'il n'a fallu que 19 j à Mbouroukou et 21 j à Mélong III pour observer ce phénomène.

\section{Conclusion}

Sur jeunes plants de pomelo Marsh à Nyombé, les inoculations ont abouti à des décolorations qui n'ont pas toutes évolué en nécroses, puisque certaines ont disparu progressivement avec le vieillissement de la feuille, le limbe reprenant sa coloration normale. Ce phénomène de rétablissement pourrait s'expliquer par la présence de conditions écologiques défavorables à la cercosporiose en dessous de 200 m d'altitude.

L'observation des plants inoculés dans différents contextes écologiques a permis
Figure 5.

Évolution du taux de feuilles tombées de jeunes plants d'agrumes pendant les dix semaines qui suivent leur contamination (29 mai 1995) avec un inoculum de spores de Phaeoramularia angolensis. Observations faites sur huit sites d'observation localisés à des altitudes différentes (Cameroun). 


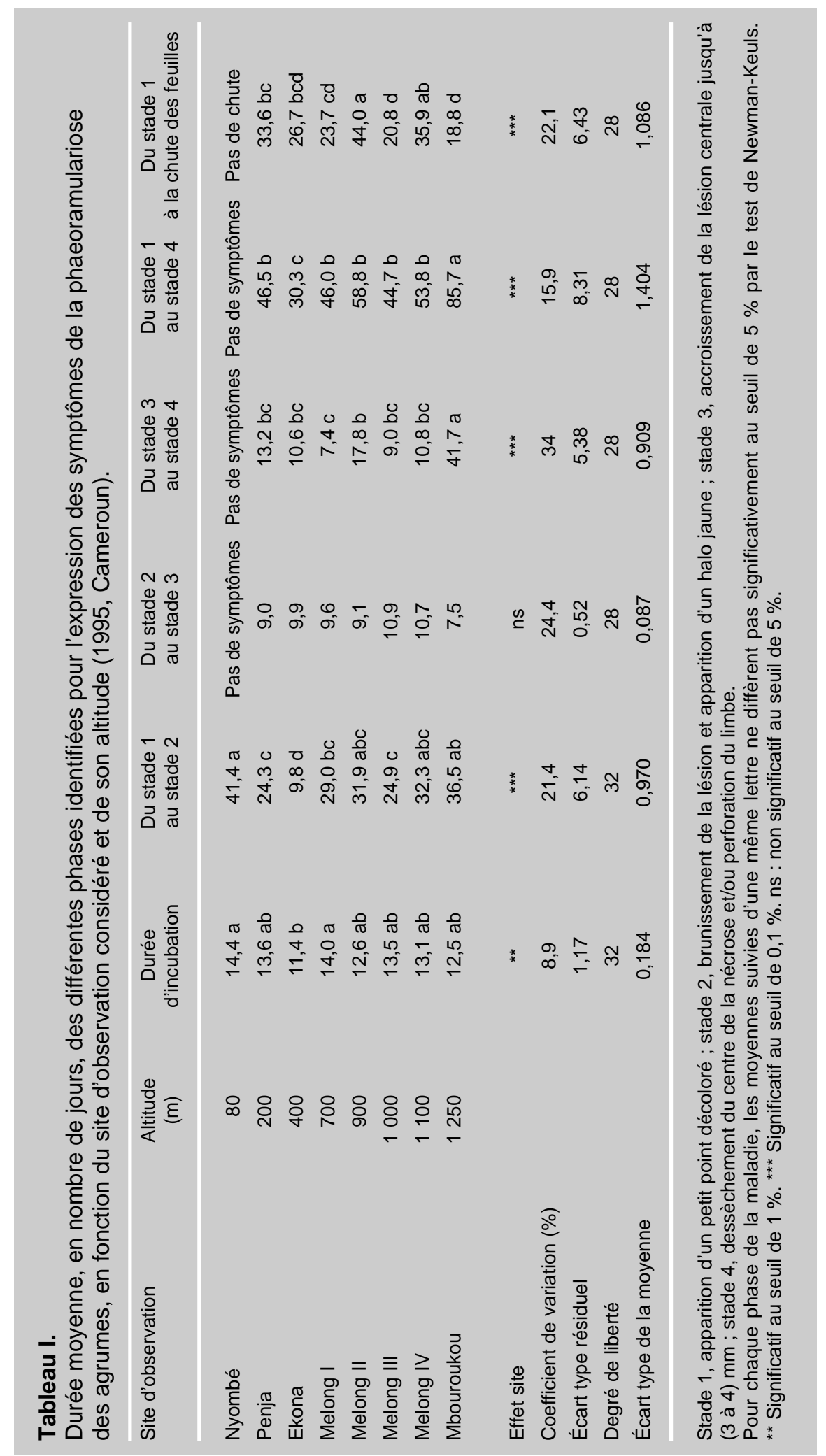


de noter l'influence du site sur l'évolution de la maladie. Le site de Nyombé $(80 \mathrm{~m})$, caractérisé par des températures moyennes supérieures à $25^{\circ} \mathrm{C}$ et des moyennes d'humidité relative inférieures à $80 \%$ (les minima étant régulièrement en dessous de $60 \%$ ) durant la période d'étude, s'est révélé assez défavorable au développement de la maladie, contrairement à d'autres sites d'altitude plus élevée (supérieure ou égale à $200 \mathrm{~m}$ ). Des moyennes de températures comprises entre (20 et 25$)^{\circ} \mathrm{C}$ avec des valeurs minimales en dessous de $20^{\circ} \mathrm{C}$ ont été observées sur les autres sites où l'expression des symptômes a été complète et rapide après les inoculations. En dehors des plants des sites de Nyombé et Penja, le pourcentage cumulé de feuilles malades a été de $100 \%$, mais l'intensité des symptômes a varié assez fortement selon les sites.

Pour l'étude de la durée des stades de développement de la maladie, aucune tendance purement liée à l'altitude du site expérimental n'a pu être dégagée. Ainsi pour la durée d'incubation par exemple, les résultats obtenus à Ekona $(400 \mathrm{~m})$ ont différé significativement de ceux obtenus à Nyombé (80 m) mais pas de ceux de Mbouroukou $(1250 \mathrm{~m})$.

Cette étude a constitué la première phase d'un travail qui sera poursuivi sur plusieurs années et dans d'autres contextes écologiques. Les résultats obtenus donnent de premiers éléments utiles qui permettront à terme de réaliser une cartographie du risque épidémique de la cercosporiose des agrumes au Cameroun.

\section{Remerciements}

Ce travail a été réalisé grâce à l'existence d'une importante logistique mise en place par l'ex-CRBP (Centre de recherches régionales sur Bananiers et plantains) dans le cadre de ses expérimentations. Nous remercions les responsables de ce centre qui nous ont permis de profiter de cette logistique. Merci à Oscar Nguidjo et Robert Dongmo pour leur collaboration.

\section{Références}

[1] De Carvalho T., Mendes O., Una cercosporiose em citrinos, Mocambique 72 (1952) 8 p.

[2] De Carvalho T., Mendes O., Una nova especie de Cercospora em Citrus sinensis Osbeck, Bol. Soc. Broteriana 2 (27) (1953) 201-202.

[3] Kirk P.M., Description of pathogenic fungi and bacteria, CMI, Set 85, No. 841850 , Kew, Surrey, England, 1986.

[4] Rey J.Y., Ducelier D., Njonga B., Maladies et ennemis des agrumes au Cameroun, Mesres-Ira, Yaoundé, Cameroun, 1986, 20 p.

[5] Bella Manga, Kuate J., Rey J.Y., Damesse F., Mimbimi Ngbwa M., Criblage variétal au champ de divers groupes d'agrumes en fonction de leur sensibilité à la cercosporiose (Cercospora angolensis de Carvalho \& Mendes), in: Cirad-Irfa, Journées Irfa Agrumes et mangues, 4-10 septembre 1991, Montpellier, France, document interne $n^{\circ} 45$, 1991.

[6] Bella Manga, Dubois C., Kuate J., Mimbimi Ngbwa M., Rey J.Y., Sensibilité à Phaeoramularia angolensis de divers groupes d'agrumes cultivés en zone forestière humide du Cameroun, Fruits 54 (3) (1999) 167-176.

[7] Rey J.Y., Njonga B., Damesse F., Fouré É., Sensibilité variétale à la cercosporiose et premiers résultats des tests fongicides dans le Centre Cameroun, in: Cirad-Irfa, Réunion annuelle 1988, Montpellier, France, document interne $\mathrm{n}^{\circ} 20,1988$.

[8] Seif A.A., Hillocks R.J., Phaeoramularia fruit and leaf spot of Citrus with special reference to Kenya, Int. J. Pest Manage. 39 (1) (1993) 44-50.

[9] Seif A.A., Hillocks R.J., Chemical control of Phaeoramularia fruit and leaf spot of citrus in Kenya, Crop Prot. 16 (2) (1997) 141-145.

[10] Kuate J., Bella Manga, Fouré É., Rey J.Y., Symptômes de la cercosporiose des agrumes due à Phaeoramularia angolensis, Fruits 49 (1) (1994) 31-36.

[11] Kuate J., Bella Manga, Rey J.Y., Fouré É., Symptômes de la cercosporiose africaine des agrumes, Symptoms of Citrus leaf spot disease, Phaeoramularia angolensis (De Carvalho \& O. Mendes) P.M. Kirk, CTA, Wageningen, The Netherlands, 1994, 19 p.

[12] Brun J., La cercosporiose des agrumes provoquée par Cercospora angolensis, Fruits 27 (7-8) (1972) 539-541. 
[13] Kuate J., Fouré É., La cercosporiose des agrumes (Cercospora angolensis) : contribution à l'étude épidémiologique dans la zone écologique de Dschang, Fruits 43 (10) (1988) 559-567.

[14] Kuate J., Bella Manga, Damesse F., Fouré É., Rey J.Y., La cercosporiose des agrumes due à Phaeoramularia angolensis. Évolution de la maladie sur fruits en zone forestière humide, Fruits 49 (2) (1994) 93-101.

[15] Kuate J., Bella Manga, Rey J.Y., Fouré É., Évolution de la cercosporiose à Phaeoramularia angolensis sur feuilles d'agrumes en zone forestière humide du Cameroun, Fruits 52 (5) (1997) 297-306.

[16] Kuate J., Cercosporiose des agrumes causée par Phaeoramularia angolensis, Cah. Agr. 7 (2) (1998) 121-129.

[17] Kuate J., La cercosporiose des agrumes au Cameroun due à Phaeoramularia angolensis (De Carvalho \& O. Mendes) P.M. Kirk. Épidémiologie, biologie in vitro du champignon et relations hôte-parasite, Carfop/Univ. Dschang, thèse, Cameroun, 1997, 184 p.

[18] Mourichon X., Peter D., Zapater M.F., Inoculations expérimentales de Mycosphaerella fijiensis Morelet sur de jeunes plantules de bananiers issues de culture in vitro, Fruits 42 (4) (1987) 195-198.

[19] Kuate J., Fouré É., Foko J., Tchio F., Ducelier D., Inoculations expérimentales de Phaeoramularia angolensis à de jeunes plants d'agrumes issus de pépinière, Fruits 52 (3) (1997) 149-157.

[20] Emechebe A.M., Brown spot disease of Citrus caused by Phaeoisariopsis sp., Ann. Appl. Biol. 97 (1981) 257-262.

[21] Ndzoumba B., Inoculations expérimentales de Cercospora angolensis sur jeunes plantules d'agrumes, Fruits 40 (3) (1985) 191-195.

[22] Loubacky V., Étude sur la cercosporiose des agrumes causée par Phaeoramularia angolensis : inoculations expérimentales et essai de mise en évidence des composés phytotoxiques, Fac. Univ. Sci. Agron. Gembloux, Mém. DEA, Gembloux, Belgique, 1997, 79 p.

[23] Seif A.A., Hillocks R.J., Reaction of some citrus cultivars to Phaeoramularia fruit and leaf spot in Kenya, Fruits 54 (5) (1999) 323-329.

[24] Seif A.A., Hillocks R.J., Some factors affecting infection of citrus by Phaeoramularia angolensis, J. Phytopathol. 146 (8/9) (1998) 385-391.

\section{La phaeoramulariosis de los cítricos en Camerún debida a Phaeoramularia angolensis: expresión parasitaria en diferentes altitudes.}

Resumen - Introducción. La phaeoramulariosis o cercosporiosis de los cítricos debida a Phaeoramularia angolensis (De Carvalho y Mendes) P.M. Kirk constituye un importante limitante de producción en África tropical. La expresión parasitaria puede variar mucho de un sitio a otro. Material y métodos. La evolución de la enfermedad en plantas jóvenes de pomelo Marsh inoculadas con una suspensión conidial se estudió en ocho sitios de altitudes diferentes $(80 \mathrm{~m}$ a $1250 \mathrm{~m})$. Se recogieron los datos de temperatura (T) y de humedad relativa (HR) máximos, medios y mínimos en los sitios durante el período de observación. Resultados y discusión. La incidencia de la enfermedad varió de manera creciente en función de la altitud. De los ocho sitios estudiados, el de Nyombé a $80 \mathrm{~m}$ de altitud (Tmed $>25{ }^{\circ} \mathrm{C}$, Tmáx $>$ $30{ }^{\circ} \mathrm{C}$ con HRmed $<80 \%$, HRmín $<60 \%$ ) se reveló muy desfavorable a la enfermedad. En cambio, la expresión de los síntomas fue completa en los otros sitios que presentan condiciones climáticas más frescas y húmedas (Tmín < $20{ }^{\circ} \mathrm{C}$ y HRmín $>60 \%$ ). Conclusión. Estos resultados constituyen los primeros datos de caracterización de las zonas de riesgo de invasión por $P$. angolensis. Estos resultados, junto con otros trabajos posteriores que contemplen distintos contextos ecológicos, permitirán establecer una cartografía del riesgo epidémico.

Camerún / Citrus paradisi / enfermedades fungosas / Pbaeoramularia angolensis / cercosporiose / sintomas / caracteristicas del sitio / altitud / temperatura / humedad relativa 